\title{
Diversity Performance of Multiantenna Systems for UMTS Cellular Phones in Different Propagation Environments
}

\author{
A. Diallo, C. Luxey, P. Le Thuc, R. Staraj, and G. Kossiavas \\ Electronics, Antennas and Telecommunications Laboratory (LEAT), University of Nice-Sophia Antipolis, CNRS UMR 6071, \\ 250 rue Albert Einstein, Bâtiment 4, Les Lucioles 1, 06560 Valbonne, France \\ Correspondence should be addressed to C. Luxey, cyril.luxey@unice.fr
}

Received 5 August 2007; Accepted 19 November 2007

Recommended by Jaume Anguera

\begin{abstract}
We present the evaluation of the diversity performance of several two-antenna systems for UMTS terminals. First, a two-antenna system with poor antenna-to-antenna isolation is described. Then, with the help of a neutralization technique, we introduced an optimized structure with high antenna-to-antenna isolation. The key parameters for an efficient diversity performance are then discussed, some of them being actually dependent on the propagation environment (uniform, indoor, outdoor, and outdoor-toindoor chosen here). All these parameters, the total efficiency, the envelope correlation coefficient, the mean effective gain, the diversity gain, and the effective diversity gain, are computed with the help of the simulated radiation patterns of both antenna systems. Next, these key parameters are measured in a reverberation chamber to validate the simulations we achieved in the uniform environment. The comparison of the performance of the two prototypes is especially showing the usefulness of using a neutralization technique for enhanced diversity antenna systems.
\end{abstract}

Copyright (C) 2008 A. Diallo et al. This is an open access article distributed under the Creative Commons Attribution License, which permits unrestricted use, distribution, and reproduction in any medium, provided the original work is properly cited.

\section{INTRODUCTION}

Today, wireless communications are part of daily life. One of the major problems to solve in a communication between a portable unit and a base station is the deep fading caused by the multipath propagating environment. To overcome this problem, one solution consists in using some diversity technique at the terminal side of the wireless link by means of gathering several antennas [1]. However, to design suitable multiantenna systems, the engineer must take into account the working environment of all these radiators in the spacelimited mobile device. The main technical challenge is to keep high isolation between the antennas; otherwise the diversity performance of the system can be drastically reduced [2]. Moreover, the handset will definitively operate in various multipath propagation environments, and the main parameters to take care of are the total efficiency, the mean effective gain (MEG), the envelope correlation coefficient $\rho_{e}$, the diversity gain (DG), and the effective diversity gain (EDG). Recently, we designed several UMTS two-antenna systems operating on a small ground plane whose size is representative of a typical mobile phone [3]. Some of these prototypes are using a neutralization technique to achieve high antenna-to- antenna isolation and to enhance total efficiency [4]. These prototypes have already been characterized in terms of $S$ parameters, total efficiency, and envelope correlation coefficient. This characterization shows that these structures have a strong potential for an efficient implementation of a diversity scheme at the terminal side of a wireless link.

In this paper, we propose to use the simulated 3D radiation patterns of these prototypes to further evaluate their diversity performance. The goal is to compute their envelope correlation, MEG, DG, and EDG in various and realistic propagation environments $[2,5-9]$. To validate these simulations, we also present some measurements in one of these environments: the uniform multipath propagation case [10]. These measurements have been done with the help of the COST 284 project, at Chalmers Institute of Technology, Sweden, where the antenna group possesses a reverberation chamber and the associated expertise needed through the Bluetest company [11-14]. The measured envelope correlation coefficients, the total efficiency, the MEG, the DG, and the EDG of all the prototypes are thus compared. The analysis of the results demonstrates the overall improvement brought by the neutralization technique and its usefulness for enhanced diversity antenna systems 


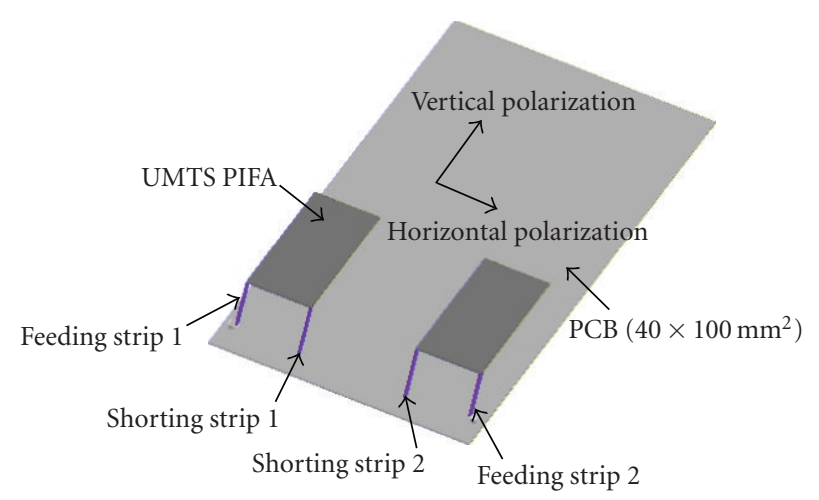

Figure 1: 3D view of the initial two-antenna system.

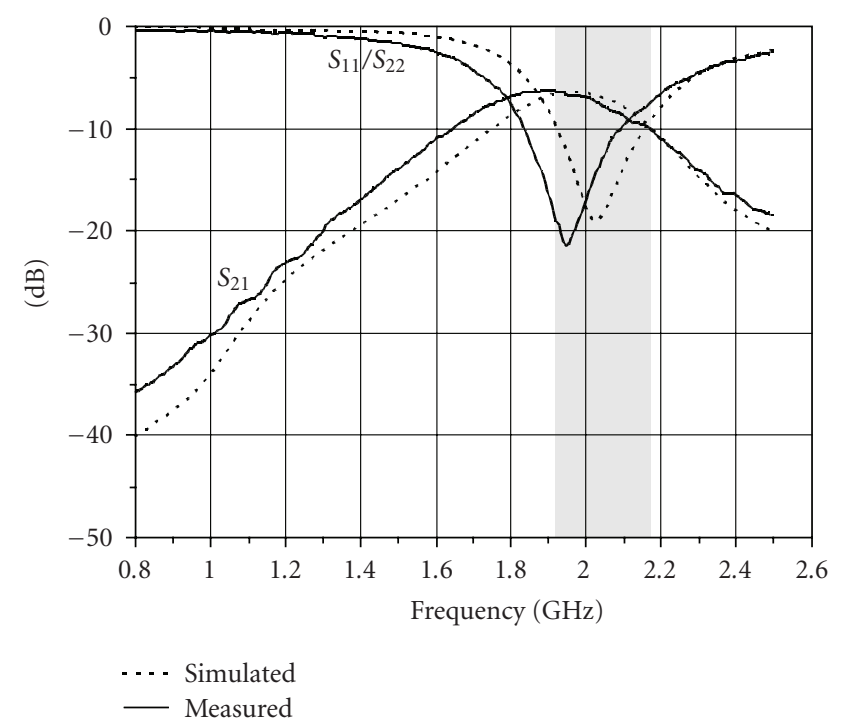

FIgURE 2: Simulated and measured $S$-parameters of the initial twoantenna system.

\section{PRESENTATION OF THE TWO-ANTENNA SYSTEMS}

The two structures of interest have been described in detail in [3]. The multiantenna systems were designed with the help of the electromagnetic software tool IE3D [15]. The initial twoantenna system is presented in Figure 1. It consists of two planar inverted-F antennas (PIFAs) positioned on the top corner of a $40 \times 100 \mathrm{~mm}^{2}$ printed circuit board $(\mathrm{PCB})$, spaced by $0.12 \lambda_{0}(18 \mathrm{~mm}$ at $2 \mathrm{GHz})$. They are fed by a metallic strip soldered to an SMA connector and shorted to the PCB by an identical strip. Each PIFA is optimized to cover the UMTS band $(1920-2170 \mathrm{MHz})$ with a return loss better than $-6 \mathrm{~dB}$. The final length is $26.5 \mathrm{~mm}$ and the final width is $8 \mathrm{~mm}$. A prototype was fabricated using a $0.3 \mathrm{~mm}$ thick nickel-silver material $\left(\mathrm{Cu}, \mathrm{Ni}, \mathrm{Zn}\right.$, conductivity $\left.\sigma=4 \times 10^{6} \mathrm{~S} / \mathrm{m}\right)$. The simulated and measured $S$-parameters of this initial structure are presented in Figure 2. Both antennas are well matched and the $S_{21}$ reaches a maximum of $-5 \mathrm{~dB}$ in the middle of the UMTS band which is considered as a poor isolation.

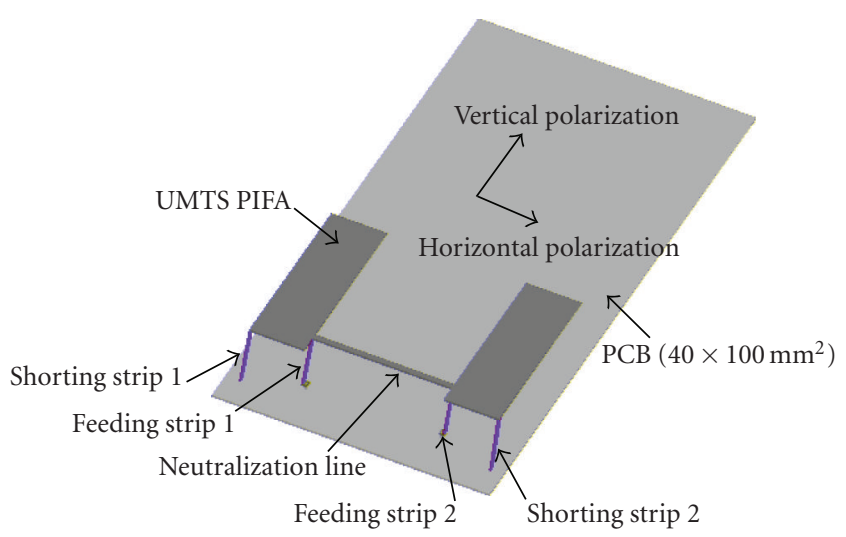

Figure 3: 3D view of the neutralized two-antenna system.

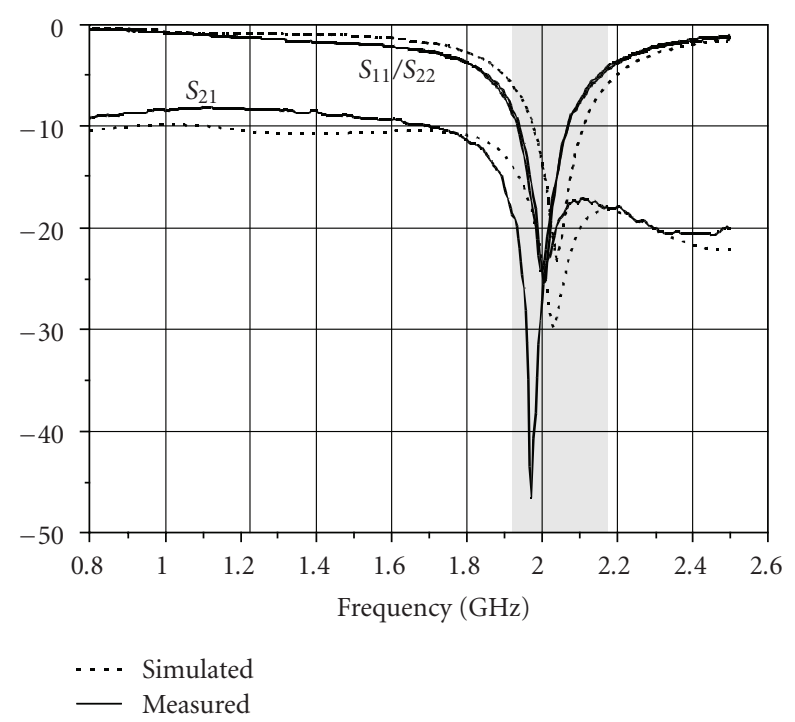

FIGURE 4: Simulated and measured $S$-parameters of the neutralized two-antenna system.

In order to improve the isolation between the radiating elements of this initial structure, a suspended line as a neutralization device was inserted between the feeding strips of the two PIFAs (Figure 3). This technique and the optimization of this line were already explained in $[3,4]$. The scattering parameters of this neutralized structure are now shown in Figure 4 . In addition to good matching, a strong improvement of antenna-to-antenna isolation is observed in the bandwidth of interest. The $S_{21}$ parameter has a deep null in the middle of the UMTS band and always remains below $-18 \mathrm{~dB}$ in this whole band: a minimum enhancement of $15 \mathrm{~dB}$ is obtained in this band when comparing this antennasystem with the initial structure. To show the benefit of having a high antenna-to-antenna isolation on the performance of multiantenna structures, the total efficiency of the two systems has been simulated with the help of IE3D (the total efficiency is defined here as the radiation efficiency + the matching and the insertion losses $[3,4])$. Both antennas of the initial system have a maximum total efficiency of $-1 \mathrm{~dB}$ 
at $2 \mathrm{GHz}$ while the ones of the neutralized structure have a total efficiency of $-0.25 \mathrm{~dB}$. Moreover, the neutralization technique induces a total efficiency improvement on the whole UMTS band. All these results seem very promising to implement some diversity in a UMTS mobile device.

\section{SIMULATED DIVERSITY PERFORMANCE IN DIFFERENT PROPAGATION ENVIRONMENTS}

It is well known that the overall performance of a mobile phone antenna can be affected by the environment in which the device is positioned [6]. Both the MEG values and the correlation of the signals arriving at the system depend on the distribution of the incoming power and the orientation of the antennas relative to this environment.

\subsection{The chosen propagation environments}

To calculate the envelope correlation coefficient $\rho_{e}$ and the MEG, we need to take into consideration the statistical propagation properties of both vertically and horizontally polarized incident radio waves. The propagation effects are defined as the power spectrum of the polarized incoming waves $P_{\theta}(\Omega)$ and $P_{\phi}(\Omega)$ expressed as statistical distribution functions $[6,7]$ :

$$
\begin{aligned}
& P_{\theta}(\Omega)=P_{\theta}(\theta, \phi)=P_{\theta}(\theta) P_{\theta}(\phi), \\
& P_{\phi}(\Omega)=P_{\phi}(\theta, \phi)=P_{\phi}(\theta) P_{\phi}(\phi) .
\end{aligned}
$$

In (1), $P_{\theta}(\theta)$ and $P_{\theta}(\phi)$ are the angular density functions in elevation; $P_{\phi}(\theta)$ and $P_{\phi}(\phi)$ are the angular density functions in azimuth. According to $[2,5-8]$, we choose some common distributions like Gaussian, Laplacian, and elliptical (Table 1). These distributions can describe some representative incoming polarized waves in elevation and/or azimuth (more complicated measurement-based angular density functions can be found in $[9,16])$. In Table $1, m_{H}$ and $m_{V}$ are the mean elevation angles of respectively the horizontally and vertically polarized wave, $\sigma_{H}$ and $\sigma_{V}$ are the horizontal and vertical standard deviations and XPR is the crosspolar discrimination (ratio of time average vertical power $(\theta)$ to the time average horizontal power $(\phi)$ of the incident field). These parameters are fully determined by a specific environment: indoor (In), outdoor (Out), and isotropic (Iso). These three environments are shaped in elevation and uniformly distributed in azimuth. The elliptical environment, shaped in both elevation and azimuth, is the one that best fits some experimental data acquired for outdoor-to-indoor communications [8]. Moreover, the constants $A_{\theta}$ and $A_{\phi}$ are determined by the following equation:

$$
\int_{0}^{2 \pi} \int_{0}^{\pi} P_{\theta}(\theta, \phi) \sin \theta d \theta d \phi=\int_{0}^{2 \pi} \int_{0}^{\pi} P_{\phi}(\theta, \phi) \sin \theta d \theta d \phi=1 .
$$

\subsection{Envelope correlation}

The independence of the fading signals incoming to the receiver of the system can be computed using the general ex- pression of $\rho_{e}$ when using the $3 \mathrm{D}$ radiation patterns as in the following equation:

$$
\rho_{e}=\frac{N}{D 1 D 2}
$$

where $N=\mid \int_{0}^{2 \pi} \int_{0}^{\pi}\left(\operatorname{XPR}_{\theta 1}(\theta, \phi) E_{\theta 2}^{*}(\theta, \phi) P_{\theta}(\theta, \phi)+E_{\phi 1}(\theta\right.$, $\left.\phi) E_{\phi 2}^{*}(\theta, \phi) P_{\phi}(\theta, \phi)\right)\left.\sin \theta d \theta d \phi\right|^{2}, D 1=\mid \int_{0}^{2 \pi} \int_{0}^{\pi}\left(\mathrm{XPR} G_{\theta 1}(\theta\right.$, ф) $\left.P_{\theta}(\theta, \phi)+G_{\phi 1}(\theta, \phi) P_{\phi}(\theta, \phi)\right) \sin \theta d \theta d \phi \mid$, and $D 2=$ $\left|\int_{0}^{2 \pi} \int_{0}^{\pi}\left(\operatorname{XPR}_{\theta 2}(\theta, \phi) P_{\theta}(\theta, \phi)+G_{\phi 2}(\theta, \phi) P_{\phi}(\theta, \phi)\right) \sin \theta d \theta d \phi\right|$, with

$$
G_{\theta n}=E_{\theta n}(\theta, \phi) E_{\theta n}^{*}(\theta, \phi)
$$

In (4), $E_{\theta n}(\theta, \phi)$ is the $\theta$ polarized complex electric field radiation pattern of the antenna number $n(n=2$ in our case).

\subsection{Mean effective gain (MEG)}

The MEG is a statistical measure of the antenna gain in a mobile environment [6]. In a particular environment, the MEG defines the power received by an antenna including the contribution of the radiation power pattern, the antenna total efficiency, and the propagation effects as in the following equation:

$$
\begin{aligned}
\mathrm{MEG}=\int_{0}^{2 \pi} \int_{0}^{\pi}( & \frac{\mathrm{XPR}}{1+\mathrm{XPR}} G_{\theta}(\theta, \phi) P_{\theta}(\theta, \phi) \\
& \left.+\frac{1}{1+\mathrm{XPR}} G_{\phi}(\theta, \phi) P_{\phi}(\theta, \phi)\right) \sin \theta d \theta d \phi .
\end{aligned}
$$

In(5), $P_{\theta}$ and $P_{\phi}$ are the angular density functions of the incident power already defined in Section 3.1. XPR represents the cross-polarization discrimination defined in the same paragraph. $G_{\theta}$ and $G_{\phi}$ are, respectively, the $\theta$ - and $\phi$ - polarized components of the antenna power gain patterns defined in (4). The MEG is normalized by a normalization of the gains as in the following equation:

$$
\int_{0}^{2 \pi} \int_{0}^{\pi}\left(G_{\theta}(\theta, \phi)+G_{\phi}(\theta, \phi)\right) \sin \theta d \theta d \phi=4 \pi
$$

\subsection{Diversity gain (DG) and effective diversity gain (EDG)}

The effectiveness of a diversity scheme is usually presented in terms of DG. It can be defined as the difference between the signal-to-noise ratio (SNR) of the combined signals of all the radiators of a multiantenna system and the SNR of the best single antenna of the structure as in the following equation:

$$
\mathrm{DG}=\left[\frac{\gamma_{C}}{\Gamma_{C}}-\frac{\gamma_{1}}{\Gamma_{1}}\right]_{P\left(\gamma_{C}<\gamma_{S} / \Gamma\right)} .
$$

In (7), $\gamma_{C}$ and $\Gamma_{C}$ are, respectively, the instantaneous and the mean SNR of the combined signal, $\gamma_{1}$ is the instantaneous SNR of the best branch, $\Gamma_{1}$ is the mean value of $\gamma_{1}$, and $\gamma_{S} / \Gamma$ is the threshold or reference level. The probability $P$ depends on both the number $M$ of the branches of the system and the 
envelope correlation $\rho_{e}$. Equation (8) gives this probability for two diversity branches when the probability of the combined signal be above the given threshold $x=\gamma_{S} / \Gamma$ [17]:

$$
\begin{aligned}
P_{\left(\gamma_{C}<x\right)=} & -\exp \left(-\frac{x}{\Gamma_{1}}\right) \\
& \cdot Q\left(\sqrt{\frac{2 x}{\Gamma_{2}\left(1-\left|\rho_{e}\right|\right)}}, \sqrt{\frac{2 \rho_{e} x}{\Gamma_{1}\left(1-\left|\rho_{e}\right|\right)}}\right) \\
& -\exp \left(-\frac{x}{\Gamma_{2}}\right) \\
& \cdot\left[1-Q\left(\sqrt{\frac{2 \rho_{e} x}{\Gamma_{2}\left(1-\left|\rho_{e}\right|\right)}}, \sqrt{\frac{2 x}{\Gamma_{1}\left(1-\left|\rho_{e}\right|\right)}}\right)\right] .
\end{aligned}
$$

In (8), $\Gamma_{1}$ and $\Gamma_{2}$ are the mean SNR value for antennas 1 and 2 (these two values are not necessary the same regarding the chosen environment and/or the orientation of the antennas in this environment), and $Q$ is the Marcum function [18]. The DG is usually given at the probability value of $1 \%$ or 99\% reliability level (Figure 5). However, the DG may not strictly express a realistic gain. In practical applications, the total efficiency of the best antenna of the system has to be taken into account. By doing so, the effective diversity gain (EDG) represents the true benefit of the diversity scheme toward a single-antenna system as in the following equation:

$$
\mathrm{EDG}=\mathrm{DG}^{*} \eta_{\text {tot. best branch }}
$$

\subsection{Computation of the diversity performance of the two structures}

The computed diversity performance of the two antenna systems in different propagation environments is given in Table 2. The initial antenna system with poor antenna-toantenna isolation is denoted "Init" and the antenna system with the neutralization line is called "Neutr." The isotropic, indoor, and outdoor cases are, respectively, called "Iso," "In," and "Out." These values have been computed at one frequency point: $2 \mathrm{GHz}$. It should be noted that the PCB, in both cases, has been vertically positioned in the environment (parallel to the xOy plane).

The first comment goes on the envelope correlation coefficient: this parameter is extremely low for both prototypes whatever the environment is. The neutralization technique does not bring any obvious improvement; nevertheless, satisfactory diversity performance is expected if this coefficient is less than 0.5 [19].

The MEG is then given for both structures, in a uniform environment. In this case and with such an antenna system orientation, the maximum achievable MEG is $-3 \mathrm{~dB}$. The MEG of the neutralized structure is always higher than the one of the initial structure. This is a consequence of the better total efficiency of the antennas of the neutralized structure. This is also the case for all the studied environments. However, the MEG is always found to be lower than the uniform case due to the spatial distribution of the incoming waves and therefore the fact that less power is intercepted by the antennas of the system. The MEG ratio has been computed for the elliptical case (two MEG values are given in Table 2 for each antenna of each prototype). This is due to the fact that in all the other cases the PCB is set vertical and the azimuth distribution of the incoming waves is kept uniform; consequently the MEG is equal for antenna 1 and antenna 2 of a same prototype and the MEG ratio is then 1. For the elliptical case, the MEG ratio remains always less than 1.12 or $0.5 \mathrm{~dB}$ which indicates that both antennas of a same prototype are equally participating to the diversity effort. There is not so much to say about the DG at the $99 \%$ reliability level; this parameter is very similar for both the initial and the neutralized structure. This comes not only from the fact that the envelope correlation coefficient is very low and almost the same for both antenna systems but also from the fact that the $3 \mathrm{D}$ radiation patterns of both structures are quite identical. Considering all the propagation environments, the DG values are ranging from $9.85 \mathrm{~dB}$ to $10.2 \mathrm{~dB}$. These values are slightly better than the one encountered in $[2,5,18]$. The combined signal was calculated considering the selection combining technique which is not only the simplest one regarding the RF chain requirements but also the one giving the lowest DG [20]. When taking into account the total efficiency of each antenna of a structure, the EDGs of the neutralized systems are always nearly $1 \mathrm{~dB}$ better than the ones of the initial prototype. This comment is valid in every propagation environment. These values are again approximately $1 \mathrm{~dB}$ better than the ones encountered in $[2,5,18]$ due to the implementation of the neutralization technique and the high total efficiency of the antennas. What is also very interesting in Table 2 is to see that even if the MEG is varying a lot from the uniform case to other environments, the EDG is not; the maximum deviation of the EDGs is less than $0.35 \mathrm{~dB}$ for both prototypes. This fact is very interesting for anyone who has the adequate facility to measure and characterize a multiantenna system in any of these environments; a relative extrapolation of the EDGs values in the other environments could be "a posteriori" done. Finally, it should be added that all these simulations were conducted at one frequency point, where the neutralized system is the most efficient; however, for UMTS handheld terminals, the diversity technique is not needed on the overall frequency band but only in the receive mode that is $60 \mathrm{MHz}$ from 2110 to $2170 \mathrm{MHz}$.

\section{REVERBERATION CHAMBER MEASUREMENTS}

The idea was to try to validate some of these simulations by measurements. Through the COST284 project, we got the possibility to perform some measurements in the Bluetest reverberation chamber (RC) of Chalmers Institute in Gothenburg, Sweden. An RC is known to reproduce an isotropic uniform multipath environment. The total efficiency of an antenna can be easily measured in the so-called RC. The procedure can be found in [11]. The measured total efficiency in $\mathrm{dB}$ versus frequency of all the antenna systems is shown in Figure 6. It can be seen two slightly different plain lines (one for each antenna) due to the fact that the fabricated prototype suffers from small asymmetries. These measurements are compared with the simulation curves obtained from IE3D (only one dotted line in this case due to a perfectly 
TABLE 1: Propagation models used to evaluate the performance of the antenna systems.

\begin{tabular}{|c|c|c|c|c|c|}
\hline Model & $\begin{array}{l}\text { Elevation/ } \\
\text { azimut }\end{array}$ & $\begin{array}{l}\text { Uniform } \\
\text { (isotropic) }\end{array}$ & Gaussian/uniform & Laplacian/uniform & Elliptical \\
\hline \multirow{11}{*}{$\begin{array}{l}\text { Statistical } \\
\text { distributions }\end{array}$} & & $P_{\theta}(\theta)=\frac{1}{4 \pi}$ & $P_{\theta}(\theta)=A_{\theta} \exp$ & $P_{\theta}(\theta)=A_{\theta} \exp$ & $P_{\theta}(\theta)=\sqrt{A_{\theta}} \frac{s_{\theta \theta}^{2}}{s_{\theta \theta}^{2}+(\sin \theta)^{2}}$ \\
\hline & & & $\times\left[-\left(\theta-\left[(\pi / 2)-m_{V}\right]\right)^{2}\right]$ & $\times\left[-\sqrt{2}\left|\theta-\left[(\pi / 2)-m_{V}\right]\right|^{2}\right]$ & \\
\hline & & & $\times\left[\overline{2 \sigma_{V}^{2}}\right]$ & $\times\left[\overline{\sigma_{V}}\right]$ & \\
\hline & & $P_{\phi}(\theta)=\frac{1}{4 \pi}$ & $P_{\phi}(\theta)=A_{\phi} \exp$ & $P_{\phi}(\theta)=A_{\phi} \exp$ & $P_{\phi}(\theta)=\sqrt{A_{\phi}} \frac{s_{\phi \theta}^{2}}{s_{\phi \theta}^{2}+(\sin \theta)^{2}}$ \\
\hline & & & $\times\left[\frac{-\left(\theta-\left[(\pi / 2)-m_{H}\right]\right)^{2}}{2 \sigma^{2}}\right]$ & $\times\left[-\frac{-\sqrt{2}}{\left|\theta-\left[(\pi / 2)-m_{H}\right]\right|^{2}}\right]$ & \\
\hline & & $P_{\theta}(\phi)=1$ & $P_{\phi}(\phi)=1$ & $P_{\theta}(\phi)=1$ & $\begin{array}{l}\text { High directivity ( } 1 / 8 \text { of } \\
\text { the sphere) }\end{array}$ \\
\hline & & $P_{\phi}(\phi)=1$ & $P_{\phi}(\phi)=1$ & $P_{\phi}(\phi)=1$ & $P_{\theta}(\phi)=\sqrt{A_{\theta}} a_{\theta 0}$ \\
\hline & & & & & $P_{\phi}(\phi)=\sqrt{A_{\phi}}\left(a_{\phi 0}-b_{\phi}|\phi|\right)$ \\
\hline & & & & & $\begin{array}{l}\text { Low directivity ( } 1 / 2 \text { of } \\
\text { the sphere) }\end{array}$ \\
\hline & & & & & $P_{\theta}(\phi)=\sqrt{A_{\theta}} \frac{s_{\theta \phi}^{2}}{s_{\theta \phi}^{2}+(\sin \phi)^{2}}$ \\
\hline & & & & & $P_{\phi}(\phi)=\sqrt{A_{\phi}} \frac{s_{\phi \phi}^{2}}{s_{\phi \phi}^{2}+(\sin \phi)^{2}}$ \\
\hline \multirow{15}{*}{$\begin{array}{l}\text { Scenario } \\
\text { parameters }\end{array}$} & & & $\mathrm{XPR}=5 \mathrm{~dB}$ & $\mathrm{XPR}=5 \mathrm{~dB}$ & \\
\hline & & & $m_{v}=10^{\circ}$ & $m_{v}=10^{\circ}$ & \\
\hline & Indoor & & $m_{H}=10^{\circ}$ & $m_{H}=10^{\circ}$ & $\mathrm{XPR}=5.5 \mathrm{~dB}$ \\
\hline & & & $\sigma_{v}=15^{\circ}$ & $\sigma_{v}=15^{\circ}$ & $s_{\theta \theta}=0.29$ \\
\hline & & & $\sigma_{H}=15^{\circ}$ & $\sigma_{H}=15^{\circ}$ & $s_{\phi \theta}=1.06$ \\
\hline & & & $\mathrm{XPR}=1 \mathrm{~dB}$ & $\mathrm{XPR}=1 \mathrm{~dB}$ & $s_{\theta \phi}=0.44$ \\
\hline & & & $m_{v}=20^{\circ}$ & $m_{v}=20^{\circ}$ & $s_{\phi \phi}=1.18$ \\
\hline & Outdoor & & $m_{H}=20^{\circ}$ & $m_{H}=20^{\circ}$ & $a_{\theta 0}=0.16$ \\
\hline & & & $\sigma_{v}=30^{\circ}$ & $\sigma_{v}=30^{\circ}$ & $a_{\phi 0}=0.70$ \\
\hline & & & $\sigma_{H}=30^{\circ}$ & $\sigma_{H}=30^{\circ}$ & $b_{\phi}=0.11$ \\
\hline & & & $\mathrm{XPR}=0 \mathrm{~dB}$ & $\mathrm{XPR}=0 \mathrm{~dB}$ & \\
\hline & & & $m_{v}=0^{\circ}$ & $m_{v}=0^{\circ}$ & \\
\hline & Isotropic* & & $m_{H}=0^{\circ}$ & $m_{H}=0^{\circ}$ & \\
\hline & & & $\sigma_{v}=\infty$ & $\sigma_{v}=\infty$ & \\
\hline & & & $\sigma_{H}=\infty$ & $\sigma_{H}=\infty$ & \\
\hline
\end{tabular}

*For Gaussian/uniform and Laplacian/uniform, the isotropic environment is an omnidirectionnal isotropic environment.

symmetrical simulation model). We can observe that all these curves are in a good agreement especially if we compare their maximum values. The small frequency shift observed between simulated and measured curves is due to the small frequency shift seen in Figures 2 and 4; it impacts directly the total efficiency. The improvement brought by the neutralization technique is clearly shown on the overall UMTS band and especially at $2 \mathrm{GHz}$; the maximum total efficiency of the neutralized antenna system is around $-0.25 \mathrm{~dB}$, whereas the one of the initial structure is less than $-0.9 \mathrm{~dB}$. These values are also in good agreement with the ones obtained with the $3 \mathrm{D}$ pattern integration, respectively, $-0.1 \mathrm{~dB}$ and $-1 \mathrm{~dB}$ (not shown in Table 2 but easily extractible from the MEG).
In the case the antenna is located in a uniform environment, the MEG is equal to the total efficiency minus $3 \mathrm{~dB}$ [9]. The measured MEG can be deduced from the previous measured total efficiency values by subtracting $3 \mathrm{~dB}$. A good agreement is then observed with the simulated values from Table 2 (uniform environment line). Moreover, to achieve a good DG, the average received power from each antenna element must be nearly equal; it corresponds to get the ratio of the MEG of the two antennas close to unity [19]. As a summary, the total efficiency and the MEG of the two structures are given in Table 3. The "Sim IE3D" values have been obtained directly from IE3D. The "Sim 3D" values have been computed using the simulated IE3D radiation patterns of the 
TABLE 2: Diversity performance of the two prototypes in different propagation environments at $2 \mathrm{GHz}$ by using their simulated 3D radiation patterns.

\begin{tabular}{lccccc}
\hline Model & & $\rho_{e}$ Init/Neutr & MEG (dB) Init/Neutr & $\begin{array}{l}\text { DG (dB) at 99\% } \\
\text { reliability Init/Neutr }\end{array}$ & $\begin{array}{l}\text { EDG (dB) at 99\% } \\
\text { reliability Init/Neutr }\end{array}$ \\
\hline Uniform & & $0.015 / 0.002$ & $-4 /-3.1$ & $10.18 / 10.2$ & $9.18 / 10.1$ \\
& Out & $0.15 / 0.12$ & $-12.3 /-11.7$ & $9.88 / 9.92$ & $8.88 / 9.82$ \\
Gaussian/uniform & In & $0.04 / 0.02$ & $-11.19 /-10.7$ & $10.11 / 10.16$ & $9.11 / 10.06$ \\
& Iso & $0.02 / 0.004$ & $-11.7 /-10.8$ & $10.17 / 10.2$ & $9.17 / 10.1$ \\
& Out & $0.16 / 0.14$ & $-12.4 /-11.7$ & $9.85 / 9.88$ & $8.85 / 9.78$ \\
Laplacian/uniform & In & $0.06 / 0.04$ & $-11.4 /-10.6$ & $10.09 / 10.12$ & $9.09 / 10.02$ \\
& Iso & $0.02 / 0.004$ & $-11.7 /-10.8$ & $10.17 / 10.2$ & $9.17 / 10.1$ \\
Elliptical & High & $0.067 / 0.012$ & $-17.44 \&-17.65 /-16.84 \&-16.84$ & $9.96 / 10.18$ & $8.96 / 10.08$ \\
& Low & $0.0016 / 0.008$ & $-19.13 \&-19.6 /-17.5 \&-17.5$ & $9.97 / 10.19$ & $8.97 / 10.09$ \\
\hline
\end{tabular}

TABLE 3: Simulated (3D pattern integration or IE3D values) and measured total efficiency of each antenna of the different antenna systems.

\begin{tabular}{lcccc}
\hline & \multicolumn{2}{c}{ Total efficiency $(\mathrm{dB})$ antenna1 } & \multicolumn{2}{c}{ Total efficiency $(\mathrm{dB})$ antenna2 } \\
& Simulated & RC & Simulated & -1 \\
Initial structure & -1 & -0.85 & -0.1 & -1.25 \\
Neutralized structure & -0.1 & -0.2 & -0.5 \\
\hline
\end{tabular}

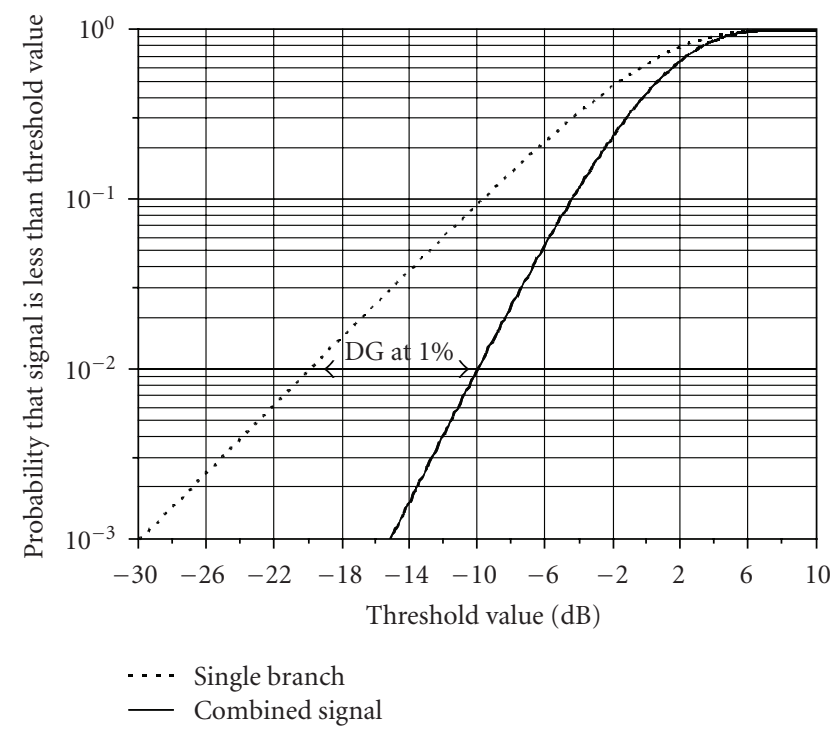

FIGURE 5: Simulated DG of the neutralized antenna-system in a uniform environment.

structures. The RC values are taken from the previous measurements. We can notice that using the neutralization line enhances the total efficiency and the MEG as expected from the previous results. The MEG ratios of the two antennas of the different prototypes have been also computed from the $\mathrm{RC}$ measurements. It is seen that the antennas have a comparable average received power; all the ratios are very close to unity: 1.12 for the initial structure and 1.07 for the neutralized one.
To measure the correlation between the antennas of our systems in the RC, each branch is connected to a separate receiver, and the outputs of the receivers are combined in such a way that the signal-to-noise ratio $S / N$ of the combined signal is larger than $S_{1} / N_{1}$ and $S_{2} / N_{2}$ of the signals in each branch. This is possible if the fading characteristics of the signal $S_{1}$ and $S_{2}$ are uncorrelated in the two branches. The measured envelope correlation coefficients of all the antenna systems are presented in Figure 7. They are compared with those obtained using (3) with the IE3D simulated complex 3D radiation patterns. All these curves are in a moderate agreement. We especially notice that the measured values are always higher than the simulated ones but the same trend shapes are observed in both cases. Moreover, the envelope correlation coefficient of all the prototypes is always largely lower than 0.15 on the whole UMTS band; significant performances in terms of diversity are thus expected.

We also extracted the DG of our two structures from the RC measurements. We can see in Figure 8 the power samples of each antenna system. Due to the measurement procedure, these measurements have been extracted over a $10 \mathrm{MHz}$ frequency band around $2 \mathrm{GHz}$, and therefore they cannot be directly compared with the results from Table 3 , computed at one frequency point. In any graph, we can observe that the curve of the combined signal with the selection combining scheme (solid line) is steeper than the two curves of each antenna element of one system taken alone (dotted lines). This is the benefit of combining the two signals received by each antenna of the structure. The diversity gain is then determined by the power level improvement at a certain probability level. In Figures 8(a), 8(b), we have chosen $1 \%$ outage probability or $99 \%$ reliability level. The difference 


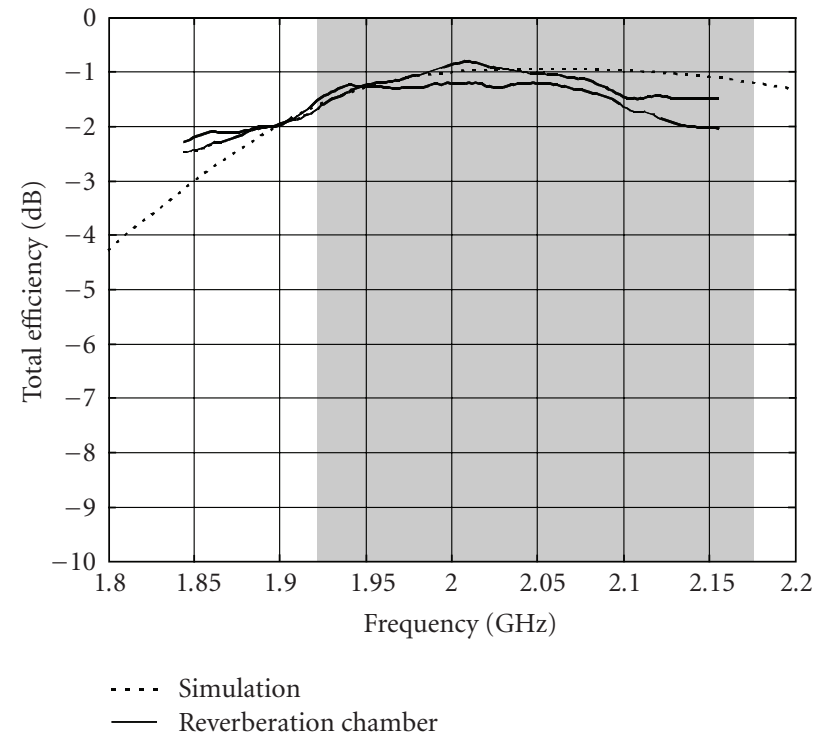

(a)

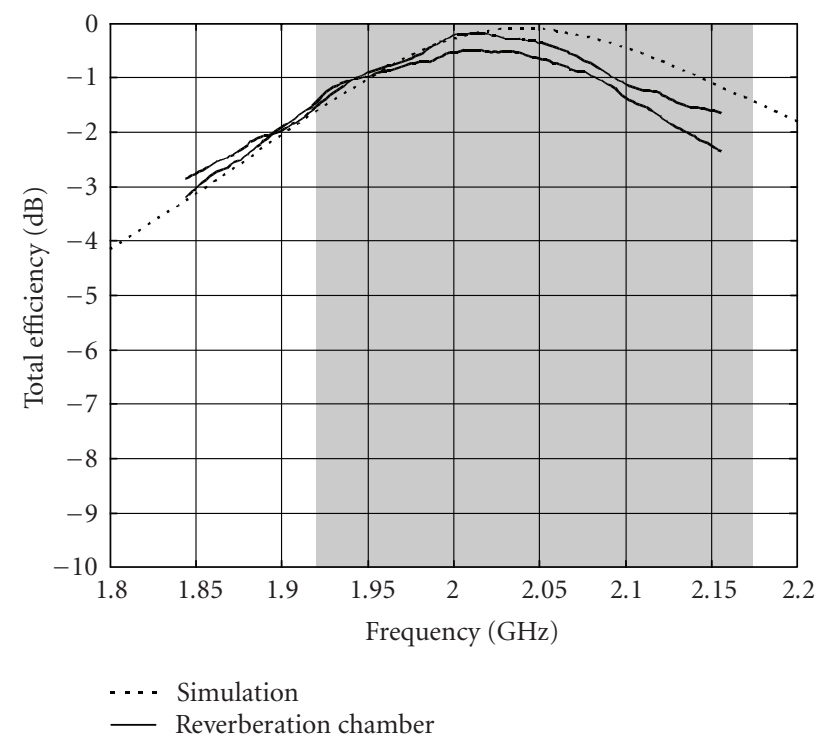

(b)

FIGURE 6: Simulated (IE3D) and measured (reverberation chamber) total efficiency of the antenna systems; (a) initial structure, (b) neutralized structure.

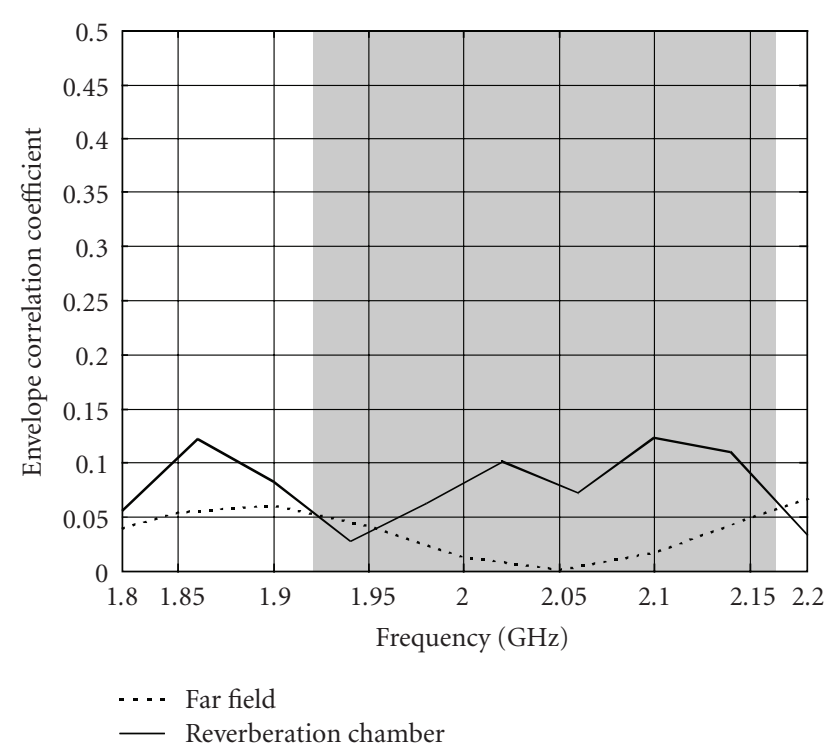

(a)

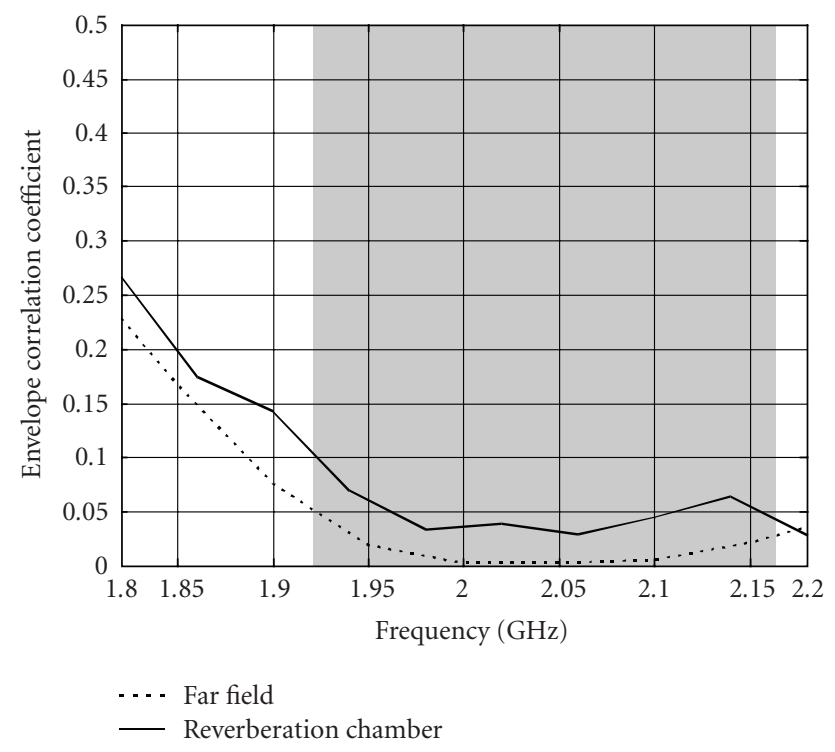

(b)

FIGURE 7: Simulated (3D patterns) and measured (reverberation chamber) envelope correlation coefficient of the two antenna systems; (a) initial structure, (b) neutralized structure.

TABLE 4: Summary of the measured and processed DGs and EDGs of all the antenna systems.

\begin{tabular}{lccc}
\hline Prototypes & Total efficiency Best branch & Diversity gain RC data/smoothed data & Effective diversity gain RC data/smoothed data \\
\hline Initial structure & $-0.85 \mathrm{~dB}$ & $7.6 \mathrm{~dB} / 8.39 \mathrm{~dB}$ & $6.85 \mathrm{~dB} / 7.74 \mathrm{~dB}$ \\
Neutralized structure & $-0.2 \mathrm{~dB}$ & $9.1 \mathrm{~dB} / 9.75 \mathrm{~dB}$ & $8.8 \mathrm{~dB} / 9.55 \mathrm{~dB}$ \\
\hline
\end{tabular}




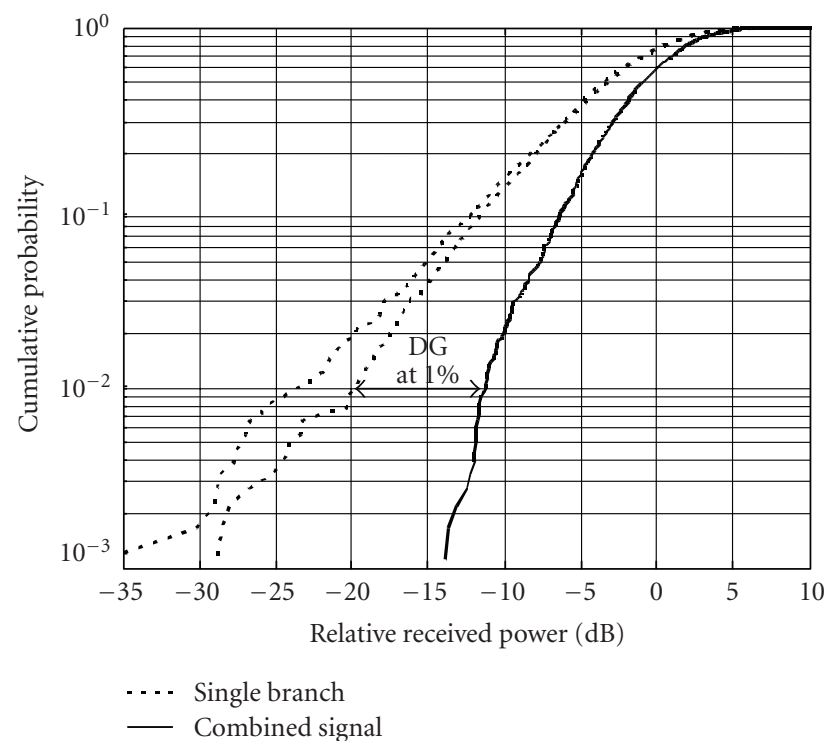

(a)

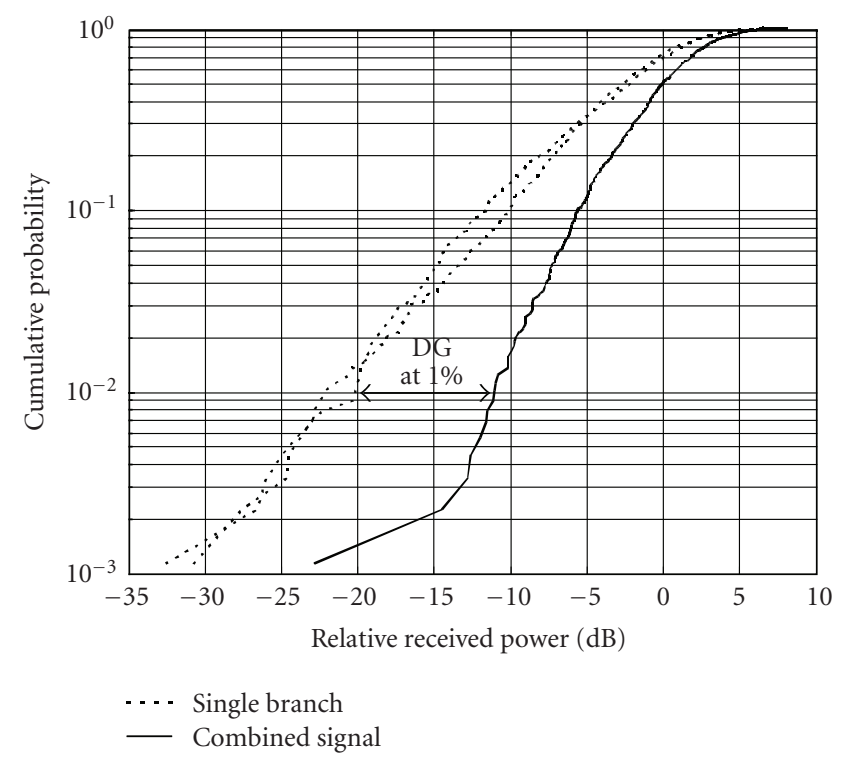

(b)

FIGURE 8: Measured cumulative probability of the two-antenna systems over a $20 \mathrm{MHz}$ bandwidth at $2 \mathrm{GHz}$; (a) initial structure, (b) neutralized structure.



(a)

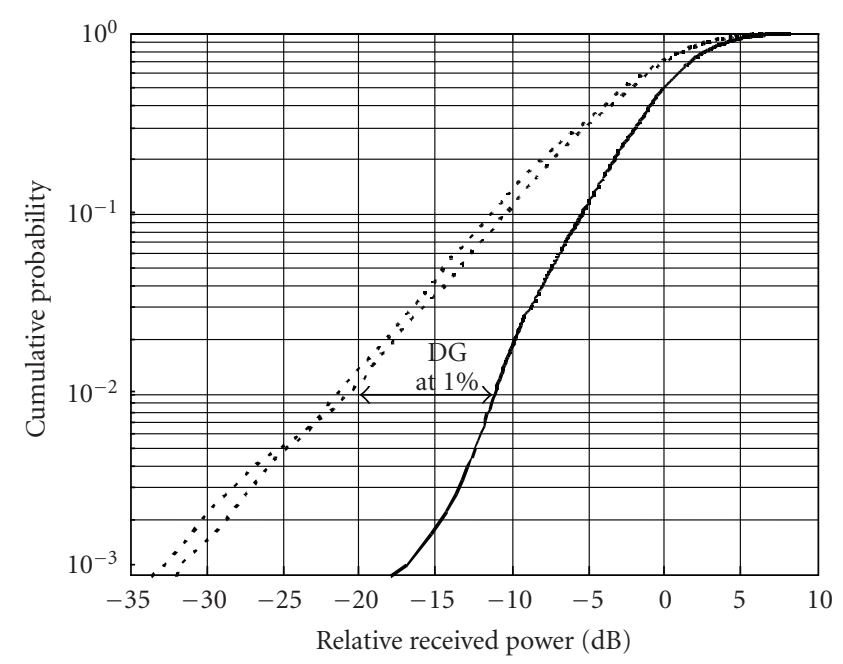

(b)

FIGURE 9: Processed cumulative probability of the two-antenna systems with the smooth function of Matlab; (a) initial structure, (b) neutralized structure.

between the strongest antenna element curve and the combined signal curve is then the DG. The power improvement is $7.6 \mathrm{~dB}$ for the initial system, whereas it is $9.1 \mathrm{~dB}$ for the neutralized system. When taking into account the total efficiency of the antennas, we can compute the EDG: $6.85 \mathrm{~dB}$ for the initial system, $8.8 \mathrm{~dB}$ for the neutralized prototype. However, by just looking at the curves in Figure 8 for low probability levels, the uncertainty on the DG values might not be neglected. The curves are in fact slightly shaky for low probability levels because of a lack of the number of the samples due to the old measurement procedure (this problem has been now solved in the new Bluestest cham- ber). The data from Figure 8 were then processed with the "smooth function" of Matlab [21] in order to evaluate the validity of our measurements. Several "smooth steps" were tried out in this operation and the new curves are presented in Figure 9. It appears that the two DGs were formerly underestimated. The new EDGs are now $7.74 \mathrm{~dB}$ and $9.55 \mathrm{~dB}$ for, respectively, the initial and the neutralized antenna systems. A summary of all these values is given in Table 4. These results are consistent with the ones of Table 2 (resp., $9.18 \mathrm{~dB}$ and $10.1 \mathrm{~dB}$ ) even if they are not directly comparable; measurements are just mean values over a $20 \mathrm{MHz}$ bandwidth while the values of Table 2 are only given at a single 
frequency point. This fact explains why the measured values are lower than the simulated ones, computed at the optimum performance of both prototypes. Nevertheless, all these results confirm the benefit of using a neutralization technique between the radiators to enhance the diversity performance.

\section{CONCLUSION}

In this paper, we presented two multiantenna systems with poor and high antenna-to-antenna isolation for diversity purpose in UMTS mobile phones. First, we computed their key parameters for diversity performance in several realistic propagation environments from their simulated 3D radiation patterns at a single frequency point: total efficiency, envelope correlation coefficient, MEG, diversity gain, and effective diversity gain. A low envelope correlation was obtained in both cases; however, the EDG of the neutralized structure is always found to be better than the one of the initial structure whatever the environment is. In addition, the maximum deviation of the EDGs considered in all the propagation environments is less than $0.35 \mathrm{~dB}$ for both prototypes. To validate some simulated results in a uniform multipath environment, we performed some measurements in the reverberation chamber of the antenna group at Chalmers Institute, Sweden. Those measurements have validated our simulations and shown that even if the envelope correlation coefficient of these systems is very low, having a multiantenna system with high antenna-to-antenna isolation will improve their total efficiency and the EDG. It also points out the usefulness of our simple neutralization solution to achieve efficient multiantenna systems at the terminal side of a wireless link for diversity applications. The fact that the RC is able to accurately characterize the EDG of a multiantenna system in a uniform environment is very interesting because with only one measurement in a specific environment, an extrapolation of the value in other environments can be "a posteriori" done if previous simulations are achieved. In this way, the RC could be accepted as a measurement standard for DG, as the anechoic chamber is for "ideal" free space line-of-sight environments.

\section{ACKNOWLEDGMENTS}

The authors express their gratitude to the chairman and the secretary of the COST284 project for providing them the opportunity to achieve a short-term scientific mission from the LEAT to Chalmers Institute. They would also like to thank Magnus Franzen and Per-Simon Kildal at, respectively, Bluetest and Chalmers Institute for their help during the measurements of their prototypes in the reverberation chamber.

\section{REFERENCES}

[1] K. Fujimoto and J. R. James, Mobile Antenna Systems Handbook, chapter 2, Artech House, Norwood, Mass, USA, 1994.
[2] T. Bolin, A. Derneryd, G. Kristensson, V. Plicanic, and Z. Ying, "Two-antenna receive diversity performance in indoor environment," Electronics Letters, vol. 41, no. 22, pp. 1205-1206, 2005.

[3] A. Diallo, C. Luxey, P. Le Thuc, R. Staraj, and G. Kossiavas, "Enhanced two-antenna structures for universal mobile telecommunications system diversity terminals," IET Microwaves, Antennas and Propagation, vol. 2, no. 1, pp. 93-101, February 2008.

[4] A. Diallo, C. Luxey, P. Le Thuc, R. Staraj, and G. Kossiavas, "Study and reduction of the mutual coupling between two mobile phone PIFAs operating in the DCS1800 and UMTS bands," IEEE Transaction on Antennas and Propagation, vol. 54, no. 11, pp. 3063-3074, 2006.

[5] Z. Ying, V. Plicanic, T. Bolin, G. Kristensson, and A. Derneryd, "Characterization of multi-channel antenna performance for mobile terminal by using near field and far field parameters," in COST 273 TD (04) (095), Göteborg, Sweden, June 2004.

[6] T. Taga, "Analysis for mean effective gain of mobile antennas in land mobile radio environments," IEEE Transactions on Vehicular Technology, vol. 39, no. 2, pp. 117-131, 1990.

[7] G. F. Pedersen and J. B. Andersen, "Handset antennas for mobile communications: integration, diversity and performance," in Review of Radio Science, chapter 5, pp. 119-137, Oxford University Press, London, UK, 1999.

[8] M. B. Knudsen and G. F. Pedersen, "Spherical outdoor to indoor power spectrum model at the mobile terminal," IEEE Journal on Selected Areas in Communications, vol. 20, no. 6, pp. 1156-1169, 2002.

[9] K. Kalliola, K. Sulonen, H. Laitinen, O. Kivekäs, J. Krogerus, and P. Vainikainen, "Angular power distribution and mean effective gain of mobile antenna in different propagation environments," IEEE Transactions on Vehicular Technology, vol. 51, no. 5, pp. 823-838, 2002.

[10] A. Diallo, C. Luxey, P. Le Thuc, R. Staraj, M. Franzén, and P.-S. Kildal, "Reverberation chamber evaluation of multi-antenna handsets having low mutual coupling and high efficiencies," in Proceedings of the 1st European Conference on Antennas and Propagation (EuCAP '06), vol. 626, Nice, France, November 2006.

[11] P.-S. Kildal and K. Rosengren, "Correlation and capacity of MIMO systems and mutual coupling, radiation efficiency, and diversity gain of their antennas: simulations and measurements in a reverberation chamber," IEEE Communications Magazine, vol. 42, no. 12, pp. 104-112, 2004.

[12] K. Rosengren and P.-S. Kildal, "Diversity performance of a small terminal antenna for UMTS," in Proceedings of Nordic Antenna Symposium, Kalmar, Sweden, May 2003.

[13] P.-S. Kildal, K. Rosengren, J. Byun, and J. Lee, "Definition of effective diversity gain and how to measure it in a reverberation chamber," Microwave and Optical Technology Letters, vol. 34, no. 1, pp. 56-59, 2002.

[14] www.bluetest.se.

[15] IE3D, “Release 11.15,” Zeland software, 2005.

[16] C. Braun, G. Engblom, and C. Beckman, "Evaluation of antenna diversity performance for mobile handsets using 3-D measurement data," IEEE Transactions on Antennas and Propagation, vol. 47, no. 11, pp. 1736-1738, 1999.

[17] M. Schwartz, W. R. Bennet, and S. Stein, Communication Systems and Techniques, vol. 4, McGraw Hill, New York, NY, USA, 1996. 
[18] V. Plicanic, "Antenna diversity studies and evaluation," M.S. thesis, Lund University, Lund, Sweden, http://www.es.lth.se/ teorel/Publications/TEAT-5000-series/TEAT-5064.pdf.

[19] S. C. K. Ko and R. D. Murch, "Compact integrated diversity antenna for wireless communications," IEEE Transactions on Antennas and Propagation, vol. 49, no. 6, pp. 954-960, 2001.

[20] Y. Gao, X. Chen, C. Parini, and Z. Ying, "Study of a dualelement PIFA array for MIMO terminals," in Proceedings of IEEE Antennas and Propagation Society International Symposium, pp. 309-312, Albuquerque, NM, USA, July 2006.

[21] http://www.mathworks.fr. 

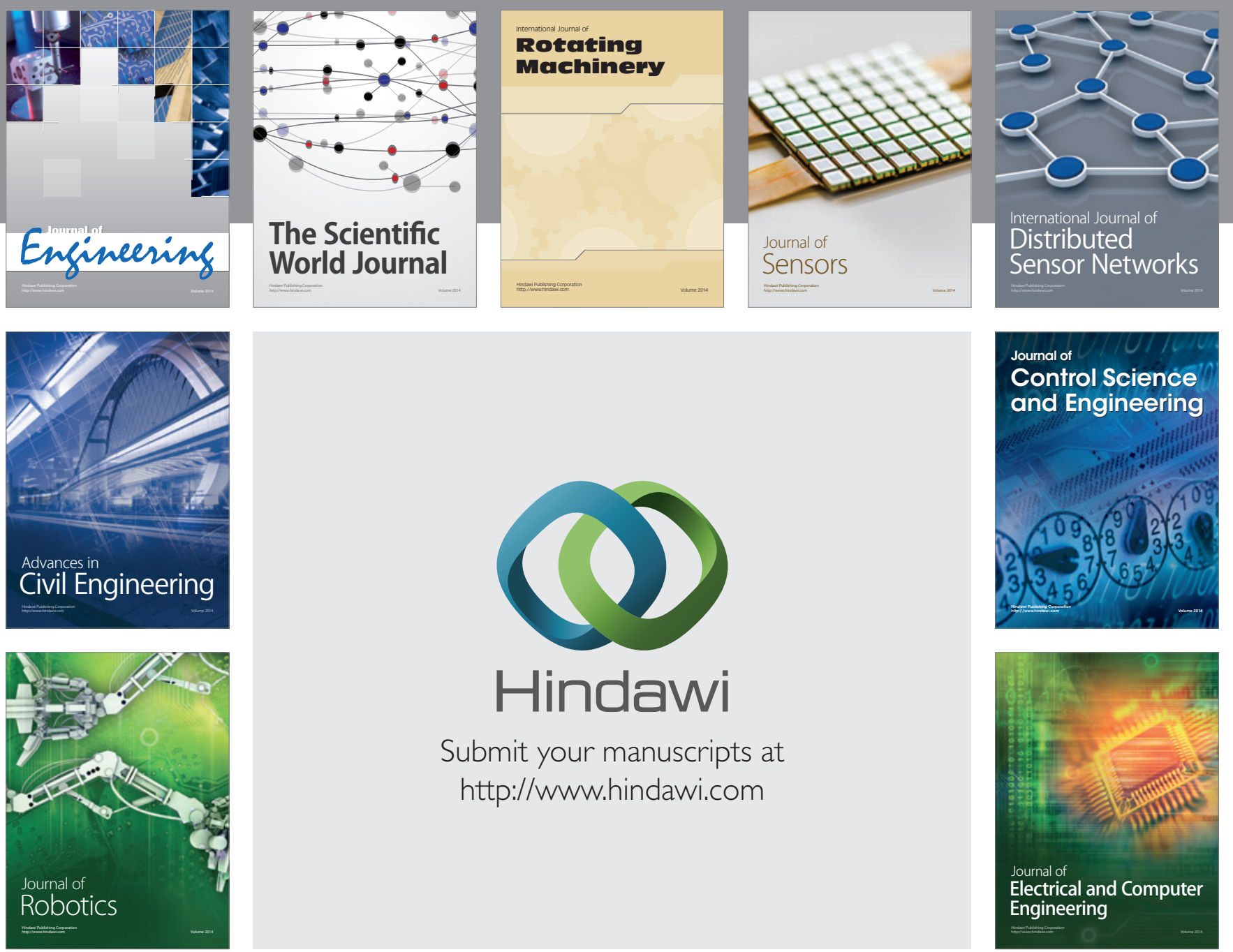

Submit your manuscripts at

http://www.hindawi.com
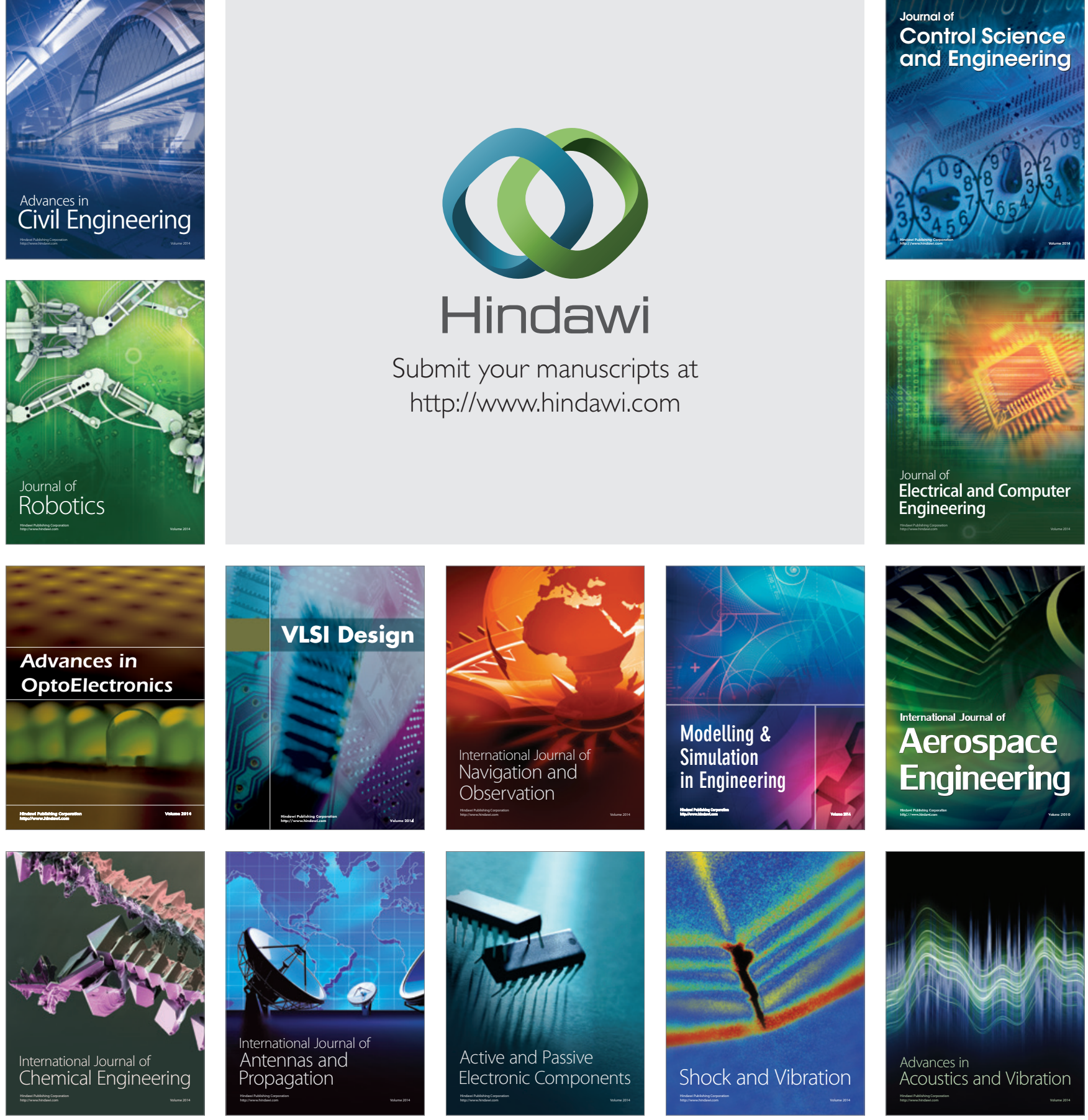